\title{
RELACIÓN DEL BLOQUEO INTERAURICULAR AVANZADO Y LA FIBRILACIÓN AURICULAR
}

\author{
ADVANCED INTERATRIAL BLOCK AND ATRIAL FIBRILLATION
}

REVISTA CONAREC 2018;33(145):149-150| DOI:10.32407/RCON/2018145/0149-0150

En el año 1979 Bayés de Luna describió los bloqueos de la conducción auricular y los clasificó como inter- e intraauriculares. Los bloqueos interauriculares (BIA) se refieren a las alteraciones de la conducción entre ambas aurículas, mientras que los bloqueos intraauriculares ocurren en la misma aurícula'. En el electrocardiograma (ECG) de superficie se observa una onda P $\geq 120 \mathrm{~ms}$, a lo cual se denominó BIA parcial; y si se agrega a la prolongación de la onda P la morfología +/- (positiva/negativa) en las derivaciones inferiores, se lo denominó BIA avanzado. También puede clasificarse como de primer grado (parcial), de segundo grado (bloqueo transitorio interauricular o aberrancia de la conducción auricular), o de tercer grado (avanzado).

Este enlentecimiento en el impulso y contracción auricular izquierda, en comparación con la derecha, genera alteraciones hemodinámicas y eléctricas. Goyal y Spodick analizaron los ecocardiogramas y ECG de pacientes con agrandamiento auricular izquierdo. Veinticuatro pacientes tenían BIA, y 16 pacientes no lo presentaban, los cuales conformaron el grupo control. Se calcularon los volúmenes de la Al (aurícula izquierda), los tiempos de aceleración de la onda A, el volumen sistólico de la Al, la fracción de eyección y la energía cinética. El resultado fue que los pacientes con BIA tuvieron iguales volúmenes de Al, significativamente más prolongados los tiempos de aceleración de la onda A y volumen sistólico, fracción de eyección y energía cinética. Se concluyó que los pacientes con BIA tienen alteración en la función contráctil de la Al, y la extensión de la disfunción está relacionada con el grado de retardo eléctrico del BIA. A su vez, sugieren que el BIA debe ser considerado como un marcador de disfunción primariamente eléctrico y con repercusión mecánica en la Al, y por lo tanto un factor de riesgo para el desarrollo de fibrilación auricular (FA) e insuficiencia cardíaca congestiva².

Esta relación entre FA y otras arritmias supraventriculares, como el aleteo auricular atípico y extrasístoles auriculares, con el BIA avanzado se evidenció en el año 1988 cuando Bayés de Luna et al. publicaron el primer trabajo³. En este estudio se compararon 16 pacientes con BIA avanzado con diferentes cardiopatías con un grupo control de iguales características clínico-ecocardiográficas, donde a todos los pacientes se les realizó registros de monitorización con Holter. El 93,7\% de los pacientes con BIA presentaron en un seguimiento a 30 meses taquicardias paroxísticas supraventriculares, mientras que solo las padecieron el 27,7\% de los pacientes del grupo control $(p<0,01)$. Al mismo tiempo, el grupo con BlA avanzado presentó mayor prevalencia de extrasístoles supraventriculares (75\%) en comparación al grupo control (16,6\%), siendo esta diferencia estadísticamente significativa $(p<0,01)^{3}$.

En el presente número de la revista, se presenta un estudio realizado entre los años 2016 y 2017 en el Hospital Privado de Rosario (Santa Fe), observacional y prospectivo, donde se incluyó a 48 pacientes internados por FA que presentaron en algún momento de su evolución reversión a ritmo sinusal, teniendo como objetivo determinar la prevalencia de BIA avanzado. Se observó que la misma fue baja (4,2\%), encontrando como limitante principal el bajo número de pacientes.

A pesar de que la prevalencia de BIA aumenta con la edad, la patogénesis exacta se desconoce. Se han propuesto diversas patologías, como la enfermedad arterial coronaria, la hipertensión arterial y la diabetes mellitus; sin embargo, ninguno de ellas mostró una correlación estadísticamente significativa con la progresión del BIA4.

Podemos concluir que el BIA representa un sustrato anátomo-eléctrico para el desarrollo de arritmias supraventriculares, como bien lo describió a fines de la década de los setenta el Dr. Bayés de Luna, lo cual se ha confirmado en numerosos estudios posteriores. Por tal motivo, esta entidad se denomina "Síndrome de Bayés"5,6. 


\section{BIBLIOGRAFÍA}

1. Conde D, Seoane L, Gysel M, Mitrione S, Bayés de Luna A, Baranchuk A. Bayés'syndrome: the association between interatrial block and supraventricular arrhythmias. Expert Rev Cardiovasc Ther 2015;13(5):541-50.

2. Goyal SB, Spodick DH. Electromechanical dysfunction of the left atrium associated with interatrial block. Am Heart J 2001;142(5):823-7.

3. Bayés de Luna A, Cladellas M, Oter R, Torner P, Guindo J, Martí V, et al. Interatrial conduction block and retrograde activation of the left atrium and paroxysmal supraventricular tachyarrhythmia. Eur Heart J 1988:9(10):1112-8.

4. Chhabra L, Devadoss R, Chaubey VK, Spodick DH. Interatrial block in the modern era. Curr Cardiol Rev 2014;10(3):181-9.

5. Conde D, Baranchuk A. Síndrome de Bayés: lo que un cardiólogo no debe dejar de saber. Rev Argent Cardiol 2014;82(3):237-9.

6. Conde D, Baranchuk A. Bloqueo interauricular como sustrato anatómico-eléctrico de arritmias supraventriculares: síndrome de Bayés. Arch Cardiol Mex 2014;84(1):32-40 